\title{
Checklist de tecamebas (Testacea) do estado de Mato Grosso do Sul, Brasil
}

\author{
Fábio Ricardo da Rosa ${ }^{1}$, Taciana Noriko Fernandes Orikassa' \\ lola Reis Lopes' \& William Marcos da Silva²
}

\author{
1. Programa de Pós-Graduação em Biologia Animal, Universidade Federal de Mato Grosso do Sul, Av. Costa e Silva, s/n²,79070-900, Caixa Postal 549, Campo Grande, \\ MS, Brasil. \\ 2. Universidade Federal de Mato Grosso do Sul, Campus Pantanal, Av. Rio Branco, 1270, 79304-902, Corumbá, MS, Brasil. Autor para correspondência \\ (wmsilvax@ig.com.br)
}

Recebido 22 novembro 2016

Aceito 6 fevereiro 2017

DOI: $10.1590 / 1678-4766 e 2017101$

ABSTRACT. Checklist of the tecamoeba (Testacea) from Mato Grosso do Sul State, Brazil. This study compiled the information on the occurrence of the testate amoeba (Protozoa: Testacea) in the Mato Grosso do Sul State. It was recorded 19 genera and 138 infrageneric taxa in the state. All these taxa were found in the Upper Paraná River basin, and only six of them were found in the Upper Paraguay River Basin. The taxonomic diversity in the Mato Grosso do Sul state was higher than São Paulo State, and comparable to the diversity recorded in the Central west and North Brazilian regions. Despite this diversity, there are few studies with no testacea and researchers living in the state.

KEYWORDS. Freshwater zooplankton, Protozoa, Alto Paraguai River Basin, Paraná River Basin, Biota-MS Program.

RESUMO. Esta síntese reuniu as informações disponíveis sobre a ocorrência de tecamebas (Protozoa: Testacea) no estado de Mato Grosso do Sul. Foram registrados 19 gêneros e 138 taxa infragenéricos no estado. Todos esses táxons foram encontrados na bacia do alto rio Paraná e apenas seis deles na bacia do alto rio Paraguai. A diversidade taxonômica foi superior à do estado de São Paulo, e comparável à diversidade das regiões Centro-Oeste e Norte. Apesar dessa diversidade, são poucos os estudos com Testacea e não há pesquisadores radicados no estado.

PALAVRAS-CHAVE. Zooplâncton de água doce, protozoários, Bacia do Alto Paraguai, Bacia do Paraná, Programa Biota-MS.

Os protozoários denominados de tecamebas pertencem à ordem Testacea, que é um grupo polifilético de organismos unicelulares eucariotos recobertos por uma carapaça formada por secreção orgânica e partículas agregadas de sílica ou calcário, a qual apresenta abertura por onde são projetados pseudópodos lobosos. Na classificação taxonômica tradicional, Testacea está inserida no filo Sarcomastigophora (amebas, flagelados e heliozoários) e na classe Sarcodina (Godinho \& ReGali-SeleGHim, 1999). Na classificação de CAVAliER-SMith (1997), as tecamebas estão inseridas no filo Rhizopoda, na classe Testacealobosea e ordem Arcellinida. E ainda, ADL et al. (2005), em sua proposta de reclassificação dos organismos eucariotos (desconsiderando a nomenclatura usual), consideraram todas as amebas testáceas integrantes do super-grupo Amoebozoa, porém diferentes gêneros aparecem no grupo Tubulinea e subgrupo Testacealobosia, grupo Flabellinea e subgrupo Thecamoebida e incertae sedis do grupo Flabellinea.

As tecamebas formam o grupo mais importante do protozooplâncton (protozoários planctônicos) de água doce, mas também ocorrem associadas ao sedimento e no perifíton desses ambientes (Godinho \& Regali-Seleghim, 1999). Protozoários têm grande importância ecológica em ambientes aquáticos, devido à variedade de nichos tróficos ocupados, são algívoros, bacterívoros, carnívoros, onívoros, osmotróficos ou mixotróficos (Godinho \& Regali-SEleghim, 1999), constituem elos alimentares para níveis tróficos superiores, transferindo a biomassa bacteriana para níveis tróficos superiores (SANDERS \& WicKHAM, 1993; BOZELLI \& HuSZAR, 2003), são estudados como opção no controle biológico de cianobactérias (Mizuta et al., 2011), atuam como remineralizadores de nutrientes no meio aquático (SHERR \& SHERR, 1994) e podem ser utilizados como bioindicadores de contaminação (PATTERSON et al., 1996) ou poluição pois estão associados à presença de matéria orgânica em decomposição (DABÉs \& VelHo, 2001).

Não há uma noção precisa da diversidade de tecamebas no mundo, apenas uma estimativa da existência de cerca de 8.000 espécies de protozoários em ambientes de água doce (Regali-Seleghim et al., 2011). No Brasil, o grupo de protozoários Testacea é o mais conhecido quanto à diversidade taxonômica (RocHA, 2003), com registros de 
pelo menos 40 gêneros e 346 táxons infragenéricos (LANSACTôHA et al., 2007).

No estado de Mato Grosso do Sul (MS) os primeiros registros de tecamebas datam do início do século XX (e.g. DADAY, 1905; WaILES, 1913; GreEN, 1975). A partir dos anos 1990 foram realizados vários estudos sobre composição e ecologia de tecamebas no estado, como LANSAC-TôHA et al. (1993; 1997), VelHo et al.(1996), VelHo \& LANSACTôHA (1996) e BonECKER et al. (1998). Tais dados foram abordados em revisões sobre a distribuição dos diferentes grupos de testáceos no Brasil (incluindo MS), realizadas por VelHo et al. (1996; 2000; 2001; 2003) e por LANSAC-TôHA et al. $(2000 ; 2001 \mathrm{a}, \mathrm{b})$. Os dados apresentados nos primeiros anos do século XXI, pelos estudos de AzEVEDo \& BONECKER (2003), BINI et al. (2003), VelHo et al. (2003), LEIPNITZ et al. (2005), MEdina JúNIOR \& RiETZER (2005) e Frutos et al. (2006) foram abordados na síntese sobre a distribuição de tecamebas no Brasil de LANSAC-TôHa et al.(2007).

Não há uma síntese do estado do conhecimento de tecamebas em Mato Grosso do Sul (MS), apesar da revisão de LANSAC-TôHA et al.(2007) apresentar também dados sobre as ocorrências de Testacea no estado. Além disso, mais recentemente, foram relatadas novas ocorrências de espécies em MS por Alves et al. (2007), LANSAC-TÔHA et al. (2008; 2009), Alves et al. (2010) e CosTA et al. (2011). Assim, o presente estudo reúne as informações disponíveis na literatura sobre as ocorrências e distribuição (considerando as bacias do Alto Rio Paraná e Alto Rio Paraguai), principais atividades de pesquisa e lacunas no conhecimento sobre tecamebas no em Mato Grosso do Sul.

\section{MATERIAL E MÉTODOS}

Os registros foram obtidos por meio de busca nas plataformas Scopus, Web of Science e no mecanismo de busca Google Acadêmico utilizando várias combinações dos termos tecamebas, Testacea, Tecamoebas, testate, amebas testáceas, testáceos, Mato Grosso do Sul, alto rio Paraná, alto rio Paraguai e Pantanal. Algumas das fontes de dados foram localizadas apenas após a consulta da bibliografia citada nas publicações, utilizando até mesmo contato direto com os autores para obter alguns manuscritos.

Para elaborar a lista dos registros de tecamebas de Mato Grosso do Sul (MS) foram compilados dados dos seguintes estudos: VelHo et al.(1996), VelHo \& LANSACTÔHA (1996), LANSAC-TÔHA et al. (1997), BONECKER et al. (1998), AzEvedo \& Bonecker (2003), Bini et al. (2003), LANSAC-TôHa et al. (2004), LEIPNITZ et al.(2005), MEDINA JúNIOR \& Rietzer (2005), Frutos et al. (2006), Alves et al.(2007), LANSAC-TÔHA et al. (2008), LANSAC-TÔHA et al. (2009), Alves et al. (2010) e Costa et al. (2011).

\section{RESULTADOS}

Foram encontrados 26 trabalhos relacionados a tecamebas de Mato Grosso do Sul. Alguns desses estudos continham enfoque ecológico, sem listagem de espécies ou citação de ocorrência (LANSAC-TôHA et al., 1993; VeLHO et al., 1999, 2003, 2004). Outros eram compilações de dados já apresentados (LANSAC-TôHA et al., 2000, 2001a,b, 2007), VelHo et al. (2000; 2001) e, nestes casos, não foram repetidos na lista geral de espécies (Tab. I). Não foram encontrados trabalhos aplicados, como de bioindicação com tecamebas ou com sistemática, taxonomia ou descrição de espécies do estado. Um dos trabalhos é uma monografia de conclusão de curso com foco em zooplâncton no qual as tecamebas foram contada em conjunto e não identificadas (AMARAL, 2009), e também não contribuindo para a listagem de espécies.

A figura 1 mostra as localidades de estudos sobre Testacea em Mato Grosso do Sul, concentrados principalmente em corpos de água ao longo das planícies de inundação do Alto Rio Paraná (na foz do rio Ivinhema, no rio Baía, lagoas conectadas ou não, canais e no próprio Rio Paraná) e de Alto Rio Paraguai (incluindo lagoas e baías no Pantanal, conectadas ou não e pontos ao longo do rio Paraguai, Negro e Miranda). Exceto nos trabalhos de Alves et al. (2010) e LEIPNITZ et al. (2005) que realizaram coletas também no sedimento bentônico e de Frutos et al. (2006), MEDINA JúNiOR \& Rietzer (2005) e Alves et al. (2010) que amostraram plâncton com rede de malha com 20,30 e 50 micrômetros, as amostras foram coletadas do plâncton, nas regiões limnética e litorânea, em alguns casos associadas a macrófitas, com rede de 68 ou 70 micrômetros e fixadas em solução de formalina a $4 \%$, algumas buferizadas, outras não. Alguns trabalhos apresentaram caráter taxonômico, mas a maioria abordou variação sazonal, relação com dados físicoquímicos ou hidrológicos, variabilidade espacial e outros temas ecológico ou limnológicos.

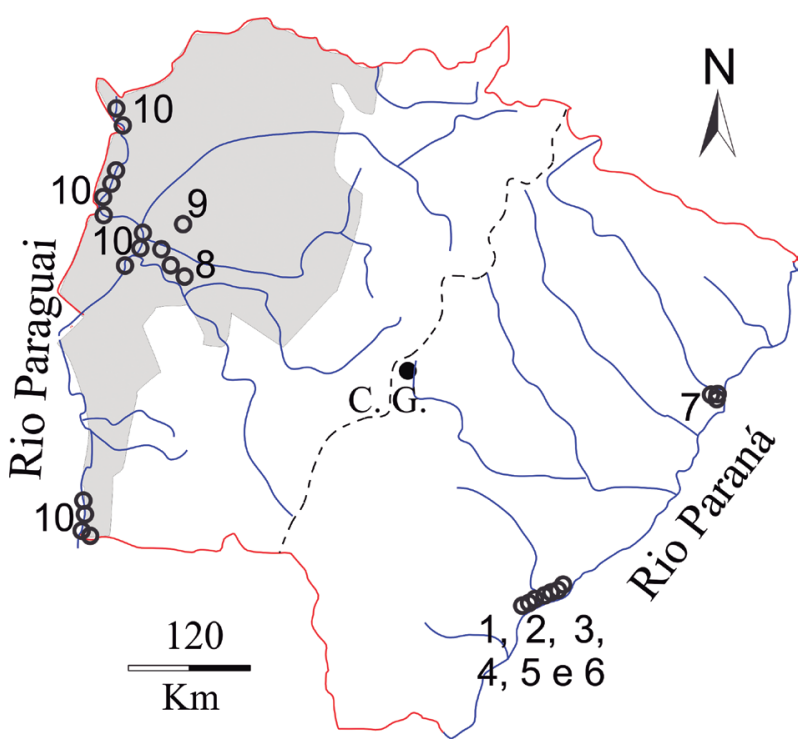

Fig. 1. Mapa com os principais rios de Mato Grosso do Sul, onde os círculos representam áreas de estudos com tecamebas, dos quais: 1, LANSAC-TôHA et al. (1997, 2004, 2008, 2009); 2, VelHo et al. (1996), VelHo \& LANSACTôHa (1996); 3, Azevedo \& Bonecker (2003); 4, Bini et al. (2003); 5, Alves et al. (2007, 2010); 6, Costa et al. (2011); 7, LeIPNITZ et al.(2005); 8, Bonecker (1998); 9, Medina-Júnior \& Rietzer (2005); 10, Frutos et al. (2006). 
Foram compilados registros de ocorrências de dez grupos (famílias), 19 gêneros e 141 taxa infragenéricos de tecamebas no estado do Mato Grosso do Sul, destes últimos, 113 espécies com algumas formas ou variedades. Todos esses táxons foram registrados na bacia do alto rio Paraná, e apenas 12 deles foram registrados na bacia do alto rio Paraguai (Tab. I).

Difflugiidae apresentou o maior número de táxons (seis gêneros e 71 táxons infragenéricos), a seguir Arcellidae (um gênero, 25 táxons infragenéricos), Centropyxidae (um gênero, 15 táxons infragenéricos), Lesquereusiidae (dois gêneros, 12 táxons infragenéricos), Trigonopyxidae (dois gêneros, sete espécies) e outras famílias menos especiosas. Estas proporções estão de acordo com o esperado, com predominância das famílias Difflugidae, Arcellidae e Centropyxidae, pois, segundo SiLva (2008), os gêneros Difflugia, Arcella e Centropyxis são os mais especiosos do Brasil, fato confirmado pelo levantamento de LANSAC-TôHA et al. (2007).

Dentre os taxa computados, 37 podem ser considerados raros, pois foram registrados em apenas um dos estudos selecionados, 19 são comuns, pois foram citados em mais da metade das publicações, enquanto que os demais apresentaram frequências intermediárias (Tab. I).

\section{DISCUSSÃO}

1. Diversidade taxonômica. A diversidade registrada no estado do Mato Grosso do Sul representa um subconjunto dos 346 táxons infragenéricos de tecamebas de ambientes aquáticos continentais brasileiros, o que inclui organismos perifíticos, bentônicos e os 282 táxons planctônicos (LANSACTôHa et al. 2007). Na lista obtida há espécies sem epíteto específico (as que constam como sp. ou spp.) que podem ser uma das espécies nomeadas ou sinônimo de uma delas. Os gêneros Arcella, Curcubitella, Difflugia e Euglypha são os de maior incerteza devido ao maior número de espécies que podem não ter sido identificadas ou, ao contrário, podem representar repetições de táxons já identificados. Heleopera, Pontigulasia, Phryganella, Trinema e Trygonopyxis pertencem a famílias com poucos gêneros, que demandam mais atenção para identificação de suas espécies.

A riqueza taxonômica do estado foi superior àquela registrada no Estado de São Paulo (67 táxons) por RegALISeleghim et al.(2011), a partir de extensa compilação dos dados de 75 corpos de água de várias sub-bacias daquele estado. Ainda, comparativamente, há registros de 21 gêneros e 87 espécies no Pantanal de Mato Grosso (Hardoim \& Heckman, 1996). Também em Mato Grosso, Oliveira \& HARDOIM (2010) registraram 80 táxons infragenéricos em um único estudo realizado sob cachoeira no município de Chapada dos Guimarães, Mato Grosso. WALKER (1982) registrou 18 gêneros e 129 morfotipos em córregos amazônicos.

Este levantamento mostrou que a região CentroOeste apresenta a maior diversidade taxonômica compilada de tecamebas no país, mesmo sendo apenas a segunda mais estudada (LANSAC-TôHA et al., 2007). Mesmo com tal diversidade potencial (e ante a importância ecológica/ limnológica do protozooplâncton), há lacunas no conhecimento sobre de tecamebas de Mato Grosso do Sul, como tratado a seguir.

\section{Abrangência, alcance e acessibilidade dos}

estudos. A distribuição agregada dos estudos ao longo das planícies de inundação do Alto Rio Paraná e Alto Rio Paraguai deixa uma extensa faixa não contemplada com pesquisas no centro do estado. Alguns ambientes menores, mesmo lóticos e/ou distantes das grandes planícies de inundação, podem apresentar protozooplâncton diversificado, como demonstrado por OlIVEIRA \& HARDOIM (2010) para poços sob cachoeiras no Cerrado de Mato Grosso. No território do Mato Grosso do Sul há abundância de riachos com ambientes permanentemente inundados, como banhados e matas paludosas, onde não foram realizadas amostragens.

Além disso, a maioria dos estudos consultados amostrou apenas tecamebas planctônicas, apesar da riqueza de espécies no sedimento e associada à macrófitas ser alta (LeIPNITZ et al., 2005), ou até maior do que no plâncton (Alves et al., 2010). Assim, pode-se esperar que a inclusão de variadas tipologias de ambientes aquáticos nos estudos e a distribuição de amostragens também na faixa central do estado devam, no mínimo, resultar em novos registros regionais de tecamebas.

Exceto nos estudos de MEdina JÚNIOR \& RIETZER (2005), Frutos et al. (2006) e Alves et al. (2010), que utilizaram redes com malhas de poro menor, é provável que as riquezas observadas em MS foram subestimadas por limitações metodológicas, pois é importante utilizar diferentes tamanhos de malhas para a coleta de zooplâncton (GodinHO \& Regali-Seleghim, 1999). Pinto-Coelho (2004) adotou poros com 50 a $65 \mu \mathrm{m}$ para amostrar protozooplâncton e GoMEs \& GodinHo (2004) consideram necessário filtrar a água em redes com malha de 10 a $25 \mu \mathrm{m}$, nos casos de espécimes de corpo mole, os quais podem mudar de formato e atravessar a rede. A rigidez da carapaça das tecamebas diminui essa possibilidade, mas as redes utilizadas na maioria dos estudos aqui compilados, com malha de 68 a $70 \mu \mathrm{m}$, apresentam poros grandes o suficiente para a evasão das tecamebas de menor porte, ou seja, podem ser seletivas. Todos os autores utilizaram solução formalina entre 4 a 5\% para preservação das amostras. Esta solução, comumente utilizada para fixar microcrustáceos, rotíferos e insetos planctônicos, causa deformação e ruptura das células de protozoários, atrapalhando o reconhecimento de características taxonômicas (Esteves, 1998; PINTO-Coelho, 2004), além de deixar as carapaças vazias e levar a não contabilização de organismos que estavam vivos antes da preservação. EsTEVES (1998) e GoDINHO \& REGALI-SELEGHIM (1999) apontaram a negligência na amostragem e preservação de tecamebas por razão do enfoque dado aos demais grupos do zooplâncton nos estudos. A julgar pelos estudos recentes levantados no estado de Mato Grosso do Sul, as metodologias ainda não foram adaptadas para tecamebas, o que constitui uma lacuna a suprimir nos próximos estudos regionais. 
Tab.I. Grupos taxonômicos e táxons infragenéricos de Testacea com ocorrências compiladas no estado do Mato Grosso do Sul, Brasil. Espécies sinônimas, indicadas por números sobrescritos após o nome válido, são relacionadas em notas após a tabela. No corpo da tabela, "x" representam registro de cada espécie em cada estudo, cujos autores são: 1, Velho et al. (1996); 2, Velho \& LansaC-TôHa (1996); 3, LANSAC-TôHa et al. (1997); 4, AzeVedo \& BonecKer (2003); 5, Bini et al. (2003); 6, LeIPNitz et al.(2005); 7, Alves et al. (2007); 8, LANSAC-TôHA et al. (2008); 9, LANSAC-TôHA et al. (2009); 10, Alves et al. (2010); 11, Costa et al. (2011); 12 LANSAC-TÔHA et al. (2004); 13, BonecKer et al. (1998); 14, MedinA-Júnior \& Rietzer (2005); 15, FRUTOS et al. (2006) (NR, número de registros; FR, frequência de registros nos estudos de cada táxon).

\begin{tabular}{|c|c|c|c|c|c|c|c|c|c|c|c|c|c|c|c|c|c|}
\hline & \multicolumn{12}{|c|}{$\begin{array}{l}\text { Bacia do } \\
\text { Alto Rio Paraná }\end{array}$} & \multicolumn{3}{|c|}{$\begin{array}{l}\text { Bacia do Alto } \\
\text { Rio Paraguai }\end{array}$} & \multirow[t]{2}{*}{ NR } & \multirow[t]{2}{*}{ FR } \\
\hline & 1 & 2 & 3 & 4 & 5 & 6 & 7 & 8 & 9 & 10 & 11 & 12 & 13 & 14 & 15 & & \\
\hline \multicolumn{18}{|l|}{ ARCELLIDAE } \\
\hline Arcella arenaria Greeff, $1866^{1}$ & & & & & & & $\mathrm{X}$ & $\mathrm{X}$ & & $\mathrm{X}$ & $\mathrm{X}$ & & & & & 4 & 0,29 \\
\hline Arcella brasiliensis Cunha, 1913 & $\mathrm{X}$ & & $\mathrm{X}$ & & & & & & & & & $\mathrm{X}$ & & & & 3 & 0,21 \\
\hline Arcella catinus Pénard, 1890 & & & & & & & & $\mathrm{X}$ & & & $\mathrm{X}$ & & & & & 2 & 0,14 \\
\hline Arcella conica (Playfair, 1917) & $\mathrm{X}$ & & $\mathrm{X}$ & $\mathrm{X}$ & & & & $\mathrm{X}$ & & $\mathrm{X}$ & $\mathrm{X}$ & $\mathrm{X}$ & & & & 7 & 0,5 \\
\hline Arcella costata Ehrenberg, 1847 & $X$ & & $\mathrm{X}$ & $\mathrm{X}$ & $\mathrm{X}$ & & & $\mathrm{X}$ & & $\mathrm{X}$ & $\mathrm{X}$ & $\mathrm{X}$ & & & & 8 & 0,57 \\
\hline Arcella crenulata Deflandre, 1928 & $\mathrm{X}$ & & $\mathrm{X}$ & & $\mathrm{X}$ & & & & & $\mathrm{X}$ & & $\mathrm{X}$ & & & & 5 & 0,36 \\
\hline Arcella dentata Ehrenberg, 1843 & $\mathrm{X}$ & & $\mathrm{X}$ & $\mathrm{X}$ & $\mathrm{X}$ & & & & & & $\mathrm{X}$ & $\mathrm{X}$ & $\mathrm{X}$ & & & 7 & 0,5 \\
\hline Arcella discoides Ehrenberg, 1843 & $\mathrm{X}$ & & $\mathrm{X}$ & $\mathrm{X}$ & $\mathrm{X}$ & & & $\mathrm{X}$ & & $\mathrm{X}$ & $\mathrm{X}$ & $\mathrm{X}$ & $\mathrm{X}$ & & & 9 & 0,64 \\
\hline Arcella gibbosa Pénard, 1890 & $\mathrm{X}$ & & $\mathrm{X}$ & & $\mathrm{X}$ & & & $\mathrm{X}$ & & $\mathrm{X}$ & $\mathrm{X}$ & $\mathrm{X}$ & & & & 7 & 0,5 \\
\hline $\begin{array}{l}\text { Arcella gibbosa var. mitriformis Deflandre, } \\
1928^{2}\end{array}$ & $\mathrm{X}$ & & & $\mathrm{X}$ & & & $\mathrm{X}$ & $\mathrm{X}$ & & $\mathrm{X}$ & $\mathrm{X}$ & & & & & 6 & 0,43 \\
\hline Arcella hemisphaerica Perty, 1852 & & & $\mathrm{X}$ & $\mathrm{X}$ & $\mathrm{X}$ & & & & & $\mathrm{X}$ & $\mathrm{X}$ & $\mathrm{X}$ & & & & 6 & 0,43 \\
\hline $\begin{array}{l}\text { Arcella hemisphaerica f. undulata } \\
\text { Deflandre, } 1928\end{array}$ & & & & & & & & & & $\mathrm{X}$ & $\mathrm{X}$ & & & & & 2 & 0,14 \\
\hline Arcella lobostoma Deflandre, 1928 & $\mathrm{X}$ & & & & & & & & & & & & & & & 1 & 0,07 \\
\hline Arcella marginata Daday, 1905 & & & & & & & & & $\mathrm{X}$ & & & & & & & 1 & 0,07 \\
\hline Arcella megastoma Pénard, 1902 & & & $\mathrm{X}$ & $\mathrm{X}$ & $\mathrm{X}$ & & & $\mathrm{X}$ & & $\mathrm{X}$ & $\mathrm{X}$ & $\mathrm{X}$ & & & & 7 & 0,5 \\
\hline Arcella mitrata Leidy, 1879 & $X$ & & $\mathrm{X}$ & $\mathrm{X}$ & $\mathrm{X}$ & & & & & $\mathrm{X}$ & $\mathrm{X}$ & $\mathrm{X}$ & & & & 7 & 0,5 \\
\hline $\begin{array}{l}\text { Arcella mitrata var. spectabilis Deflandre, } \\
1928\end{array}$ & $\mathrm{X}$ & & $\mathrm{X}$ & $\mathrm{X}$ & $\mathrm{X}$ & & & & & $\mathrm{X}$ & $\mathrm{X}$ & $\mathrm{X}$ & & & & 7 & 0,5 \\
\hline Arcella nordestina Vucetich, 1973 & $\mathrm{X}$ & & $\mathrm{X}$ & & $\mathrm{X}$ & & & & & $\mathrm{X}$ & & $\mathrm{X}$ & & & & 5 & 0,36 \\
\hline Arcella rota Daday, 1905 & $\mathrm{X}$ & & $\mathrm{X}$ & & $\mathrm{X}$ & & & & & $\mathrm{X}$ & & & & & & 4 & 0,29 \\
\hline $\begin{array}{l}\text { Arcella rotunda var. aplanata Deflandre, } \\
1928\end{array}$ & & & & & & & & & & $\mathrm{X}$ & & & & & & 1 & 0,07 \\
\hline Arcella vulgaris Ehrenberg, 1830 & & & $\mathrm{X}$ & & & & & & & & & $\mathrm{X}$ & $\mathrm{X}$ & & & 3 & 0,21 \\
\hline Arcella vulgaris f. elegans Deflandre, 1928 & & & & & $\mathrm{X}$ & & $\mathrm{X}$ & $\mathrm{X}$ & & $\mathrm{X}$ & $\mathrm{X}$ & & & & & 5 & 0,36 \\
\hline $\begin{array}{l}\text { Arcella vulgaris f. undulata Deflandre, } \\
1928\end{array}$ & $\mathrm{X}$ & & $\mathrm{X}$ & & $\mathrm{X}$ & & & & & $\mathrm{X}$ & $\mathrm{X}$ & $\mathrm{X}$ & & & & 6 & 0,43 \\
\hline $\begin{array}{l}\text { Arcella vulgaris var. penardi Deflandre, } \\
\quad 1928\end{array}$ & $\mathrm{X}$ & & $\mathrm{X}$ & & $\mathrm{X}$ & & & $\mathrm{X}$ & & & & $\mathrm{X}$ & & & & 5 & 0,36 \\
\hline $\begin{array}{l}\text { Arcella } \text { spp. } \\
\text { CENTROPYXIDAE }\end{array}$ & & & & & $\mathrm{X}$ & & & & & & & $\mathrm{X}$ & & $\mathrm{X}$ & $\mathrm{X}$ & 4 & 0,29 \\
\hline Centropyxis aculeata (Ehrenberg, 1838) & $\mathrm{X}$ & & $\mathrm{X}$ & $\mathrm{X}$ & $\mathrm{X}$ & $\mathrm{X}$ & & $\mathrm{X}$ & & & $\mathrm{X}$ & $\mathrm{X}$ & $\mathrm{X}$ & & & 9 & 0,64 \\
\hline $\begin{array}{l}\text { Centropyxis aculeata var. tropica } \\
\text { Deflandre, } 1929\end{array}$ & $\mathrm{X}$ & & & & & & & & & $\mathrm{X}$ & & & & & & 2 & 0,14 \\
\hline Centropyxis aerophila Deflandre, 1929 & & & & $\mathrm{X}$ & & $\mathrm{X}$ & & $\mathrm{X}$ & & $\mathrm{X}$ & $\mathrm{X}$ & & & & & 5 & 0,36 \\
\hline $\begin{array}{l}\text { Centropyxis aerophila var. sphagnicola } \\
\text { Deflandre, } 1929\end{array}$ & & & & & & & $\mathrm{X}$ & $\mathrm{X}$ & & $\mathrm{X}$ & & & & & & 3 & 0,21 \\
\hline Centropyxis aplanata Deflandre, 1929 & & & & & & & & $\mathrm{X}$ & & & & & & & & 1 & 0,07 \\
\hline Centropyxis cassis (Wallich, 1864) & & & & $\mathrm{X}$ & $\mathrm{X}$ & & & $X$ & & & $\mathrm{X}$ & $\mathrm{X}$ & & & & 5 & 0,36 \\
\hline Centropyxis constricta (Ehrenberg, 1841) & & & & & $\mathrm{X}$ & $\mathrm{X}$ & & $\mathrm{X}$ & & $\mathrm{X}$ & $\mathrm{X}$ & $\mathrm{X}$ & & & & 6 & 0,43 \\
\hline Centropyxis discoides (Pénard, 1890) & $\mathrm{X}$ & & $\mathrm{X}$ & $\mathrm{X}$ & $\mathrm{X}$ & $\mathrm{X}$ & & $X$ & & $\mathrm{X}$ & & $\mathrm{X}$ & & & & 8 & 0,57 \\
\hline Centropyxis ecornis (Ehrenberg, 1841) & $\mathrm{X}$ & & $\mathrm{X}$ & $\mathrm{X}$ & $\mathrm{X}$ & & & X & & $\mathrm{X}$ & $\mathrm{X}$ & & $\mathrm{X}$ & & & 8 & 0,57 \\
\hline Centropyxis gibba Deflandre, 1929 & & & & & & & $\mathrm{X}$ & $\mathrm{X}$ & & $\mathrm{X}$ & $\mathrm{X}$ & & & & & 4 & 0,29 \\
\hline Centropyxis hemisphaerica (Walles, 1913) & & & & $\mathrm{X}$ & & & & & & & & & & & & 1 & 0,07 \\
\hline Centropyxis hirsuta Deflandre, 1929 & $\mathrm{X}$ & & $\mathrm{X}$ & $\mathrm{X}$ & $\mathrm{X}$ & & & $\mathrm{X}$ & & $\mathrm{X}$ & $\mathrm{X}$ & $\mathrm{X}$ & & & & 8 & 0,57 \\
\hline Centropyxis marsupiformis (Wallich, 1864) & $\mathrm{X}$ & & $\mathrm{X}$ & $\mathrm{X}$ & $\mathrm{X}$ & & & $\mathrm{X}$ & & $\mathrm{X}$ & $\mathrm{X}$ & $\mathrm{X}$ & & & & 8 & 0,57 \\
\hline Centropyxis platystoma (Pénard, 1902) & & & & $\mathrm{X}$ & $\mathrm{X}$ & $\mathrm{X}$ & & $\mathrm{X}$ & & $\mathrm{X}$ & $\mathrm{X}$ & $\mathrm{X}$ & & & & 7 & 0,5 \\
\hline $\begin{array}{l}\text { Centropyxis spinosa }(\text { Cash, } 1905)^{3} \\
\text { DIFFLUGIIDAE }\end{array}$ & & & & & & & $\mathrm{X}$ & $X$ & & $\mathrm{X}$ & $\mathrm{X}$ & & & & & 4 & 0,29 \\
\hline $\begin{array}{l}\text { Cucurbitella crateriformis Gauthier-Lievrè } \\
\text { \& Thomas, } 1960\end{array}$ & & $\mathrm{X}$ & & & $\mathrm{X}$ & & & $\mathrm{X}$ & & & $\mathrm{X}$ & $\mathrm{X}$ & & & & 5 & 0,36 \\
\hline $\begin{array}{l}\text { Cucurbitella dentata Gauthier-Lievrè \& } \\
\text { Thomas, } 1960\end{array}$ & & & & $\mathrm{X}$ & & & & & & & & & & & & 1 & 0,07 \\
\hline $\begin{array}{l}\text { Cucurbitella dentata f. crucilobata } \\
\text { Gauthier-Lievrè \& Thomas, } 1958\end{array}$ & & & & & & & & $\mathrm{X}$ & & $\mathrm{X}$ & $\mathrm{X}$ & & & & & 3 & 0,21 \\
\hline $\begin{array}{l}\text { Cucurbitella dentata f. quinquelobata } \\
\text { Gauthier-Lievrè \& Thomas, } 1960\end{array}$ & & X & $\mathrm{X}$ & X & $\mathrm{X}$ & & & $\mathrm{X}$ & & $\mathrm{X}$ & $\mathrm{X}$ & $\mathrm{X}$ & & & & 8 & 0,57 \\
\hline
\end{tabular}


Tab.I. Cont

Cucurbitella dentata f. trilobata Gauthier-

Lievrè \& Thomas, 1958

Cucurbitella dentata var. simplex Gauthier-

Lievrè \& Thomas, 1958

Cucurbitella madagascarensis Gauthier-

Lievrè \& Thomas, 1960

Cucurbitella mespiliformis Pénard, 1902

Cucurbitella mespiliformis var. africana $\mathrm{f}$.

trilobata Gauthier-Lievrè, 1958

Cucurbitella neotropicalis Dioni, 1970

Curcubitela sp.

Difflugia acuminata Ehremberg, 1838

Difflugia acuminata var. inflata Pénard,

1899

Difflugia acuminata var. magna Deflandre,

1926

Difflugia acutissima Deflandre, 1931

Difflugia amphoralis Hopkinson, 1909

Difflugia amphoralis var. cornuta

Hopkinson, 1909

Difflugia amphoralis var. globosa

Gauthier-Lièvre \& Thomas, 1958

Difflugia angulostoma Gauthier-Lièvre \&

Thomas, 1958

Difflugia bicruris Gauthier-Lièvre \&

Thomas, 1958

Difflugia capreolata Gauthier-Lièvre \&

Thomas, 1958

Difflugia corona Wallich, 1864

Difflugia corona var. ecornis Gauthier-

Lievrè \& Thomas, 1958

Difflugia corona var. tuberculata Vucetich, 1978

Difflugia correntina Vucetich, 1978

Difflugia curvicaulis Pénard, 1899

Difflugia difficillis Thomas, 1955

Difflugia echinulata Pénard, 1911

Difflugia elegans Pénard, 1890

Difflugia fragosa Hempel, 1898

Difflugia globularis (Wallich, 1864)

Difflugia globulosa Dujardin, 1837

Difflugia gramen Pénard, 1902

Difflugia helvetica var. multilobata

Gauthier-Lievrè \& Thomas, 1958

Difflugia kempnyi Stepánek, 1953

Difflugia lanceolata Penard, 1890

Difflugia limnetica Levander, 1900

Difflugia lingula Pénard, 1911

Difflugia lithophila Pénard, 1902

Difflugia lobostoma Leidy, 1879

Difflugia lobostoma var. cornuta Gauthier-

Lievrè \& Thomas, 1958

Difflugia lobostoma f. multilobata

Gauthier-Lievrè \& Thomas, 1958

Difflugia lobostoma var. tuberosa Gauthier-

Lievrè \& Thomas, 1958

Difflugia cf. mamaliformis Gauthier-Lievrè

\& Thomas, 1958

Difflugia microclaviformis (Kourov, 1925)

Difflugia mitriformis Gauthier-Lievrè \&

Thomas, 1958

Difflugia multidentata Dioni, 1970

Difflugia muriculata Gauthier-Lievrè \&

Thomas, 1958

Difflugia muriformis Gauthier-Lievrè \&

Thomas, 1958

Difflugia muriformis f. crucilobata

Gauthier-Lievrè \& Thomas, 1958

\begin{tabular}{|c|c|c|c|c|c|c|c|c|c|c|c|c|c|}
\hline & & & & & & $X$ & & $X$ & $X$ & & & 3 & 0,21 \\
\hline & & & & $X$ & & & & & & & & 1 & 0,07 \\
\hline$X$ & $X$ & & & & & & & $\mathrm{X}$ & $\mathrm{X}$ & & & 4 & 0,29 \\
\hline \multirow[t]{2}{*}{$X$} & $X$ & & $X$ & $X$ & & $X$ & & $X$ & $X$ & $X$ & & 8 & 0,57 \\
\hline & & & & & & & $X$ & & & & & 1 & 0,07 \\
\hline \multirow[t]{3}{*}{$X$} & & & $X$ & & & & & & & $X$ & & 3 & 0,21 \\
\hline & $X$ & & & & & & & & & $X$ & & 1 & 0,07 \\
\hline & $X$ & $X$ & $X$ & $X$ & & $X$ & & $X$ & $\mathrm{X}$ & $X$ & & 8 & 0,57 \\
\hline$X$ & & & & & & $X$ & $X$ & & & & & 3 & 0,21 \\
\hline \multirow[t]{8}{*}{$X$} & $X$ & & $X$ & & & & & & & $X$ & & 4 & 0,29 \\
\hline & & & & & $X$ & $X$ & & $X$ & & & & 3 & 0,21 \\
\hline & & & & & & & & $X$ & & & & 1 & 0,07 \\
\hline & & & & & & $X$ & & & $X$ & & & 2 & 0,14 \\
\hline & & & & & $X$ & $X$ & & & $X$ & & & 3 & 0,21 \\
\hline & & & & & $X$ & & & $X$ & & & & 2 & 0,14 \\
\hline & & & & $X$ & $X$ & & & $\mathrm{X}$ & & & & 3 & 0,21 \\
\hline & & & & $X$ & & & & $X$ & & & & 2 & 0,14 \\
\hline \multirow[t]{2}{*}{$X$} & & $X$ & $X$ & $X$ & & $X$ & & $X$ & $X$ & $X$ & & 8 & 0,57 \\
\hline & & & & & & & $X$ & & & & & 1 & 0,07 \\
\hline$X$ & $X$ & $X$ & $X$ & & & & & $\mathrm{X}$ & & $X$ & & 6 & 0,43 \\
\hline$X$ & $X$ & & & & & $X$ & & $X$ & & $X$ & & 5 & 0,36 \\
\hline \multirow[t]{2}{*}{$\mathrm{X}$} & $\mathrm{X}$ & & $X$ & & & $X$ & & & & $X$ & $X$ & 6 & 0,43 \\
\hline & & & & & $X$ & & & $X$ & & & & 2 & 0,14 \\
\hline$X$ & X & & $X$ & & & & & & & $X$ & & 4 & 0,29 \\
\hline \multirow[t]{4}{*}{$X$} & $X$ & & $X$ & & & & & X & & $X$ & & 5 & 0,36 \\
\hline & & & & & & $X$ & $X$ & & & & & 2 & 0,14 \\
\hline & & & & & & X & $X$ & X & $X$ & & & 4 & 0,29 \\
\hline & & & & & & $X$ & & & & & & 1 & 0,07 \\
\hline \multirow[t]{2}{*}{$X$} & $\mathrm{X}$ & $X$ & $X$ & & & $X$ & & $X$ & $X$ & $X$ & & 8 & 0,57 \\
\hline & & & & & $X$ & & & & & & & 1 & 0,07 \\
\hline \multirow[t]{4}{*}{$X$} & $X$ & & & $X$ & & & & & & $X$ & & 4 & 0,29 \\
\hline & & & $X$ & $X$ & & $X$ & & & & $X$ & & 4 & 0,29 \\
\hline & & & & & & $X$ & & $X$ & $X$ & & & 3 & 0,21 \\
\hline & & & & $X$ & & $X$ & $X$ & & & & & 3 & 0,21 \\
\hline$X$ & $X$ & & $X$ & & & $X$ & & $X$ & $X$ & $X$ & & 7 & 0,5 \\
\hline \multirow[t]{2}{*}{$X$} & $\mathrm{X}$ & $X$ & $\mathrm{X}$ & $X$ & & $X$ & & $X$ & $X$ & $X$ & $X$ & 10 & 0,71 \\
\hline & & & & & $\mathrm{X}$ & $X$ & & $X$ & $X$ & & & 4 & 0,29 \\
\hline \multirow[t]{6}{*}{$X$} & $X$ & & X & & & $X$ & & $X$ & $X$ & $X$ & & 7 & 0,5 \\
\hline & & & & & $X$ & $X$ & & $X$ & $X$ & & & 4 & 0,29 \\
\hline & & & & & & & & & & $\mathrm{X}$ & & 1 & 0,07 \\
\hline & & & & & X & $X$ & & $\mathrm{X}$ & $\mathrm{X}$ & & & 4 & 0,29 \\
\hline & & & & $X$ & & & & & & & & 1 & 0,07 \\
\hline & & & & & & & $X$ & & & & & 1 & 0,07 \\
\hline$X$ & & & $X$ & & & X & & & X & $\mathrm{X}$ & & 5 & 0,36 \\
\hline \multirow[t]{2}{*}{$X$} & $X$ & $X$ & $X$ & & & $X$ & & $X$ & $X$ & $X$ & & 8 & 0,57 \\
\hline & & & & & $\mathrm{X}$ & X & & $X$ & $X$ & & & 4 & 0,29 \\
\hline
\end{tabular}


Tab.I. Cont.

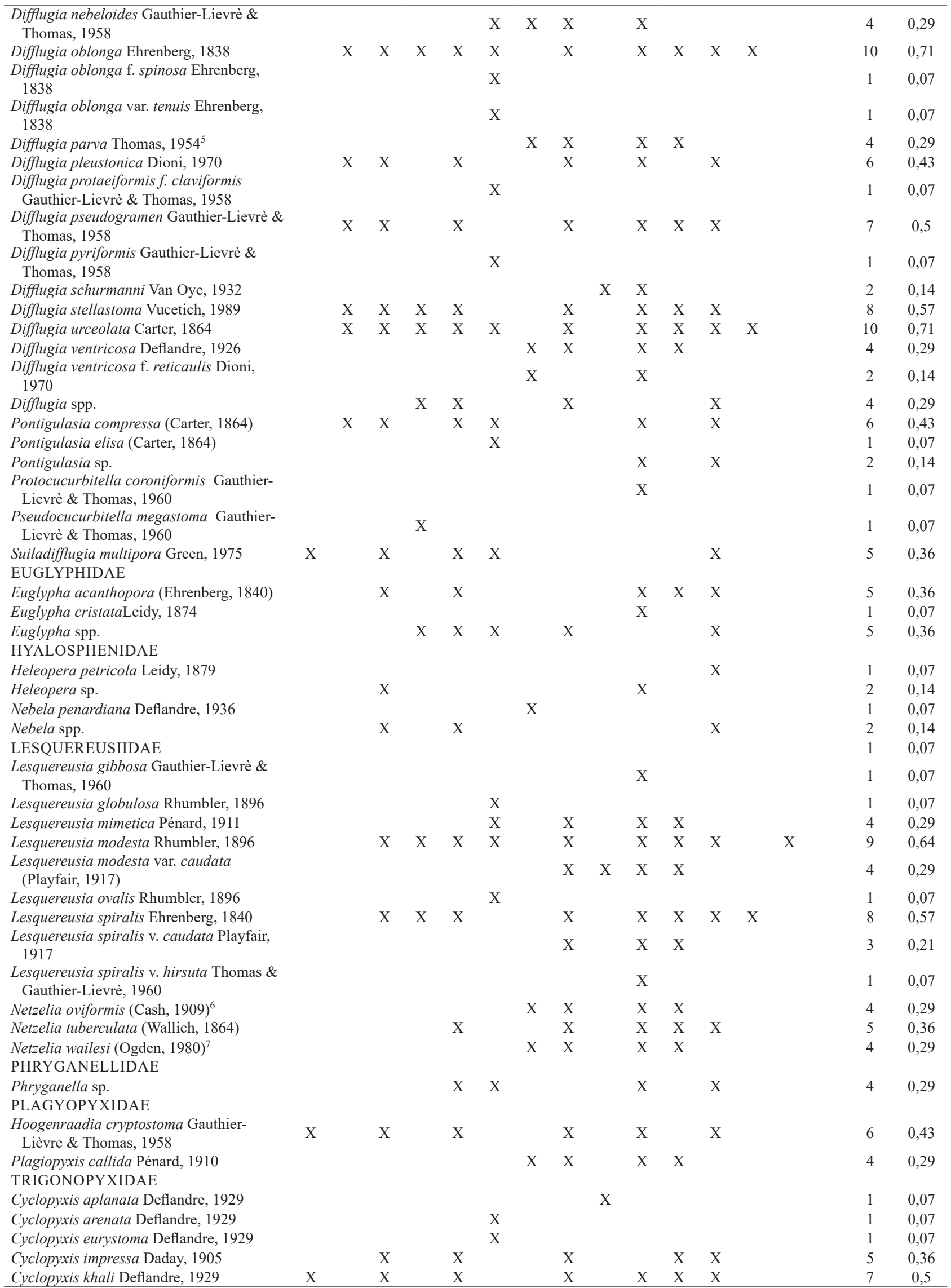


Tab.I. Cont.

\begin{tabular}{|c|c|c|c|}
\hline Cyclopyxis sp. & $\mathrm{X}$ & 1 & 0,07 \\
\hline $\begin{array}{l}\text { Trygonopyxis sp. } \\
\text { TRINEMATIDAE }\end{array}$ & $\mathrm{X}$ & 1 & 0,07 \\
\hline Trinema sp. & $\mathrm{X}$ & 1 & 0,07 \\
\hline
\end{tabular}

${ }^{1}$ Arcella aureola Maggi, 1888; A. microstoma Pénard, $1890 ;{ }^{2}$ Arcella. mitrata var. angulata; A. vulgaris Laidy, $1879 ;{ }^{3}$ Centropyxis aculeata var. spinosa Cash, 1905; ${ }^{4}$ Difflugia oblonga var. microclaviformis Kourov, $1925 ;{ }^{5}$ Difflugia oblonga var. parva Thomas, 1954; D. piriformes var. parva Chardez \& Decloitre, 1973; ${ }^{6}$ Difflugia oviformis Cash, $1909 ;{ }^{7}$ Difflugia tuberculata var. minor Wailes, 1912; D. oviformis Netzel, 1972; D. wailesi Ogden, 1980.

Vinte e um dos estudos sobre tecamebas no estado são artigos publicados, dois são capítulos de livros e um consta como texto em anais de congressos, o que indica boa acessibilidade ao conhecimento gerado. Não foram localizados manuscritos inéditos, nem mesmo monografias e outros trabalhos acadêmicos durante esta revisão.

\section{Perspectivas de pesquisa para os próximos 10}

anos. Há algumas divergências taxonômicas e sistemáticas em tecamebas, como indefinições entre espécies e variedades (MADRID, 2006), e o status incertae sedis das famílias, cuja resolução poderia ser auxiliada por técnicas de biologia molecular e genética, como a inclusão de Arcellenidae em Amebozoa feita por NiCOLAEV et al. (2005), e redescrição de espécies acrescendo características morfométricas (MADRID, 2006). Além disso, condições ambientais podem conduzir mudanças estruturais nas tecas o que dificulta a identificação das espécies pela formação de variedades (SILVA, 2008), o que representam grandes desafios à pesquisa mostrando a urgência da formação de taxonomistas para o grupo.

É necessária a ampliação dos levantamentos em outras bacias do estado além das estudadas nas proximidades dos rios Paraná e Paraguai, incluindo-se principalmente corpos de água de baixa ordem, seus ambientes adjacentes e áreas temporariamente inundáveis do Pantanal. Sanadas as lacunas de distribuição biogeográfica de Testacea no estado, há grande demanda por estudos sobre a ecologia de espécies, estratégias de vida, comparações entre meso e micro-habitats, tais como região litorânea e limnética de lagos, nascentes e córregos. Associações funcionais entre tecamebas e com outros grupos como a já elaborada para o fitoplâncton por REYNOLDS et al. (2002), também não são explorados. Isso exigiria uma grande compilação dos dados já disponíveis e comparações com os trabalhos de regiões temperadas.

A consolidação do conhecimento taxonômico, biogeográfico e ecológico permitirá a utilização de tecamebas em biomonitoramento. A utilização desse grupo como bioindicadores ainda é incipiente no Brasil (e.g. AMARAL, 2009), mas é possível encontrar muitos trabalhos internacionais disponíveis sobre o tema. Tecamebas são usadas como indicadores de alterações ambientais (PATTERSON et al., 1996), de grau trófico em ambientes aquáticos (Escobar et al., 2005) e em paleoecologia (AsIOLI et al.,1996). No Brasil, estudos sobre tratamentos de efluentes apresentam gêneros como Arcella, Centropyxis e Euglypha (e.g. BENTO et al., 2005) como presente em lodos e tanques de tratamento com boa depuração, o que pode ser revertido para a elaboração de um índice trófico com tecamebas, caso as espécies e seus níveis de tolerância sejam conhecidos. Alguns trabalhos associaram ambientes eutróficos a altas densidades de protozoários (e.g. Gomes \& Godinho, 2003; Araúso \& Costa, 2007) tomando-as como indicativo de alteração ambiental.

\section{Principais grupos de pesquisa e acervos de} Testacea do Mato Grosso do Sul. A maioria das pesquisas realizadas em MS foi efetuada por pesquisadores de instituições de outros estados, dentre as quais:

Núcleo de Pesquisa em Limnologia, Ictiologia e Aquicultura (NUPELIA), Pós-Graduação em Ecologia de Ambientes Aquáticos Continentais, Universidade Estadual de Maringá (PR), onde Cláudia Costa Bonecker, Ciro Yoshio Joko, Erica Mayumi Takahashi, Fábio Amodêo Lansac-Tôha, Geziele Mucio Alves, Fábio de Azevedo, Leandro Junio Fulone, Leandro M. Bini e Luiz Felipe Machado Velho foram os autores frequentes de publicações com registros de tecamebas em MS (citados em ordem alfabética).

No Centro de Ecología Aplicada del Litoral e da Facultad de Ciencias Exactas, Físicas y Naturales, Universidad Nacional del Nordeste, em Corrientes, Argentina, estão radicados Santa Margarita Frutos, Alicia Susana Guadalupe Poi Neiff e Juan José Neiff, que realizaram amostragens de zooplâncton ao longo do rio Paraguai, incluindo tecamebas no trecho sul-matogrossense desse rio.

Radicado em Mato Grosso do Sul, Paulino Medina Júnior, Universidade Federal da Grande Dourados (UFGD), realizou pesquisas com zooplâncton em MS, especialmente em lagoas do Pantanal.

Na Universidade Federal de Mato Grosso (UFMT), há um grupo com pesquisas sobre tecamebas do alto rio Paraguai em andamento, liderado por Edna Lopes Hardoim.

Não há acervos formais de protozoários no Brasil (Godinho \& Regali-Seleghim, 1999; Rocha, 2003) e a única coleção de lâminas de protozoários, do Laboratório de Protistologia do Instituto de Biologia da Universidade Federal do Rio de Janeiro, inclui apenas ciliados (REGALISELEGHIM et al., 2011).

Há amostras de zooplâncton em acervos regionais, grande parte fixada em formaldeído $4 \%$, que mantém, em geral, apenas as carapaças das tecamebas. Os principais locais com amostras de zooplâncton depositado em MS são: Laboratório de Microbiologia Ambiental da UFMS campus de Campo Grande; Laboratório de Ecologia da UFMS campus do Pantanal - Corumbá; Laboratório de Recursos hídricos da UFMS, campus de Aquidauana; laboratórios da UEMS na Unidade de Coxim e na Unidade de Dourados e Laboratório 
de Biodiversidade, Ecologia e Conservação de Ecossistemas Aquáticos (UNIDERP), Campo Grande, MS.

Fora do estado, há material depositado no Laboratório de Plâncton e no Laboratório de Ecologia de Microorganismos Aquáticos, ambos do Departamento de Ecologia e Biologia Evolutiva da Universidade Federal de São Carlos, SP (RochA, 2003) e na Coleção Zoológica da Universidade Estadual de Maringá, PR, parte do qual coletado em MS (LANSAC-TôHA et al., 2008).

5. Considerações Finais. A diversidade de tecamebas no estado do Mato Grosso do Sul é alta se comparada ao protozooplâncton do estado de São Paulo (relativamente bem estudado), ou mesmo quando comparada a outros estudos na região Centro-Oeste, que por enquanto apresenta a maior riqueza de espécies.

É provável que essa diversidade seja ainda maior, que ocorram mais registros de táxons, considerando a distribuição agregada das pesquisas já realizadas (ao longo da planície de inundação dos rios Paraná e Paraguai), a variedade de tipologias de ambientes aquáticos ainda não exploradas e a utilização quase exclusiva de redes com poros de $68 \mu \mathrm{m}$ a $70 \mu \mathrm{m}$, uma limitação metodológica capaz de subestimar a riqueza de protozooplâncton regional.

Apesar dessa diversidade e da importância limnológica do protozooplâncton, não há pesquisadores dedicados e radicados no estado, e são poucos os estudos realizados com Testacea em comparação aos demais grupos do plâncton dulcícola. Tais estudos são concentrados nas planícies de inundação do Alto Rio Paraná e do Alto Rio Paraguai e abrangem basicamente grandes rios e lagoas. $\mathrm{O}$ registro da distribuição e ecologia do grupo no estado reflete esse esforço amostral e os dados sobre variação sazonal e espacial e taxonômicos também estão restritos as mesmas áreas de estudo.

Agradecimentos. Agradecemos a Fundação de Apoio ao Desenvolvimento do Ensino, Ciências e Tecnologia do Estado de Mato Grosso do Sul (Fundect) e a Superintendência de Ciências e Tecnologia do Estado de Mato Grosso do Sul (Sucitec/MS) pelo convite de participação neste fascículo especial da Iheringia, Série Zoologia e o suporte financeiro para sua publicação. Agradecemos ao Dr. Fábio Amodêo Lansac-Tôha e Dra. Cláudia Costa Bonecker, do Núcleo de Pesquisas em Limnologia e Aquicultura (NUPELIA), pelo envio de manuscritos de acesso mais restrito.

\section{REFERÊNCIAS BIBLIOGRÁFICAS}

Adl, M. S.; Simpson, A. G. B.; Farmer, M. A.; Andersen, R. A.; Anderson, O. R.; Barta, J.; Bowser, S. S.; Brugerolle, G.; Fensome, R. A.; FredericQ, S.; James, T. Y.; Karpov, S.; Kugrens, P.; Krug, J.; Lane, C.; Lewis, L. A.; Lodge, J.; Lynn, D. H.; MAnN, D. G.; Mccourt, R. M.; Mendoz, L.; Moestrup, O.; Mosley-Standridge, S. E.; Nerad, T. A.; Shearer, C. A.; Smirnov, A. V.; Sitegel, F. \& Taylor, F. J. R. 2005. The new higher level classification of eukaryotes with emphasis on the taxonomy of protists. Journal of Eukaryotic Microbiology 52:399-451.

Alves, G. M.; Lansac-Tôha , F. A.; Velho, L. F. M.; Joko, C. Y. \& Costa, D. M. 2007. New records of testate lobose amoebae (Protozoa, Arcellinida) for the Upper Paraná River floodplain. Acta Limnologica Brasiliensia 19(2): 175-195.

Alves, G. M.; Velho, L. F. M.; Simões, N. R. \& Lansac-Tôha , F. A. 2010. Biodiversity of testate amoebae (Arcellinida and Euglyphida) in different habitats of a lake in the Upper Paraná River floodplain. European Journal of Protistology 46:310-318.
AmARAL, D. C. 2009. Análise do estado de conservação de duas nascentes do córrego criminoso (bacia do rio Taquari, Coxim, MS) com ênfase na comunidade zooplanctônica como bioindicadora. Dissertação de Mestrado, Fundação Universidade Estadual de Mato Grosso do Sul, Coxim.

AraúJo, M. F. F. \& Costa, I. A. S. 2007. Comunidades microbianas (bacterioplâncton e protozooplâncton) em reservatórios do semi-árido brasileiro. Oecologia brasiliensis 11(3):422-432.

Asioli, A.; Medioli, F. S. \& Patterson, R. T. 1996. Thecamoebians as a tool for reconstruction of paleoenvironments in some Italian lakes in the foothills of the southern alps (Orta, Varese and Candia). Journal of Foraminiferal Research 26(3):248-263.

Azevedo, F. \& Bonecker, C. C. 2003. Community size structure of zooplanktonic assemblages in three lakes on the upper Paraná River floodplain, PR-MS, Brazil. Hydrobiologia 505:147-158.

Bento, A. P.; Sezerino, P. H.; PhilipPi, L. S.; Reginatto, V. \& LaPolli, F. R. 2005. Caracterização da microfauna em estação de tratamento de esgotos do tipo lodos ativados: um instrumento de avaliação e controle do processo. Engenharia Sanitária e Ambiental 10(4):329-338.

Bini, L. M.; Velho, L. F. M. \& LansaC-TôHa, F. A. 2003. The effect of connectivity on the relationship between local and regional species richness of testate amoebae (Protozoa, Rhizopoda) in floodplain lagoons of the Upper Paraná River, Brazil. Acta Oecologica 24:145-151.

Bonecker, C. C.; Lansac-TôHa, F. A. \& Bini, L. M. 1998. Composition of zooplankton in different environments of the Mato Grosso do Sul, Brazil. In: VIII Seminário Regional de Ecologia. São Carlos, UFSCar, p.1123-1135.

Bozelli, R. L. \& Huszar, V. L. M. 2003. Comunidades fito e zooplanctônicas continentais em tempo de avaliação. LimnoTemas. Sociedade Brasileira de Limnologia. Disponível em <http://www.ablimno.org.br/arquivos/ limnotemas/Limnotemasv3.pdf>.

Cavalier-Smith, T. 1997. Amoeboflagellates and mitochondrial cristae in eukaryote evolution: megasystematics of the new protozoan subkingdoms Eozoa and Neozoa. Archiv für Protistenkunde 147(34):237-258.

Costa, D. M.; Alves, G. M.; Velho, L. F. M. \& LansaC-TôHa, F. A. 2011. Species richness of testate amoebae in different environments from the upper Paraná river floodplain (PR/MS). Acta Scientiarum, Biological Sciences 33(3):263-270.

DabÉs, M. B. G. S. \& VelHo, L. F. M. 2001. Assemblage of testate amoebae (Protozoa, Rhizopoda) associated to aquatic macrophytes stands in a marginal lake of the São Francisco river floodplain, Brazil. Acta Scientiarum, Biological Sciences 23(2):299-304.

DAdAY, E. 1905. Untersuchungen über die Süsswasser Mikrofauna Paraguaya. Zoologica 18(44):1-342.

Escobar, J.; Martínez, J. I. \&PARra, L. N. 2005. Thecamoebians (Testaceous Rhizopods) from a tropical lake: La Fe reservoir, Antioquia, Colombia. Caldasia 27(2):293-298

Esteves, F. A. 1998. Fundamentos de Limnologia. 2ed. Rio de Janeiro, Interciência. 602p.

Frutos, S. M.; Poi De NeifF, A. S. G. \& NeifF, J. J. 2006. Zooplankton of the Paraguay River: a comparison between sections and hydrological phases. Annales de Limnologie-International Journal of Limnology 42(4):277-288.

Godinho, M. J. L. \& Regali-Seleghim, M. H. 1999. Diversidade no Reino Protista: protozoários de vida livre. In: Joly, C. A. \& Bicudo, C.E.M. orgs. Biodiversidade do Estado de São Paulo, Brasil. São Paulo, Biota Fapesp, p. 83-91.

Gomes, E. A. T. \& GodinHo, M. J. L. 2003. Structure of the protozooplankton community in a tropical shallow and eutrophic lake in Brazil. Acta Oecologica 24:S153-S161.

Gomes, E. A. T. \& Godinho, M. J. L. 2004. Bactérias e protozoários em ambientes aquáticos: amostragem e análise. In: Bicudo, C. E. M. \& Bicudo, D. C. orgs. Amostragem em Limnologia.São Carlos, Rima, p.121-132.

Green, J. 1975. Freshwater ecology in the Mato Grosso, central Brazil. IV. Associations of testate Rhizopoda. Journal of Natural History 9:545-560.

Hardoim, E. L. \& HeCKMAN, C. W. 1996.The seasonal succession of biotic communities in wetlands of the tropical wet and dry climatic zone: IV. Free living sarcodines and ciliates of the Pantanal of Mato Grosso, 
Brazil. Revue der gesamten Hydrobiologie und Hydrographie Internationale 81(3):367-384.

Lansac-TôHa, F. A.; Bonecker, C. C. \&Velho, L. F. M. 2004. Composition, species richness and abundance of the zooplankton community. In: Thомaz, S. M. et al. ed. The upper Paraná river and its floodplain: physical aspects, ecology and conservation. Leiden, Backhuys Publishers, p.145-190.

LansaC-TôHa, F. A.; Bonecker, C. C.; Velho, L. F. M. \& Lima, A. F. 1997. Composição, distribuição e abundância da comunidade zooplanctônica. In: Vazzoler, A. E. A. M. et al. eds. A planície de inundação do alto rio Paraná: aspectos físicos, biológicos e socioeconômicos. Maringá, Eduem, p.117-155.

LansaC-TôHa, F. A.; Lima, A. F.; Thomaz, S. M. \& Roberto, M. C. 1993 Zooplâncton de uma planície de inundação do rio Paraná. II. Variação sazonal e influência dos níveis fluviométricos sobre a comunidade. Acta Limnologica Brasiliensia 8:42-55.

Lansac-Tôha, F. A.; Velho, L. F. M.; Zimmermann-Callegari, M. C. \& BoneCKER, C. C. 2000. On the occurrence of testate amoebae (Protozoa, Rhizopoda) in Brazilian inland waters. I. Family Arcellidae. Acta Scientiarum, Biological Sciences 22(2):355-363.

LansaC-Tôha, F. A.; Velho, L. F. M.; Zimmermann-Callegari, M. C.; BONECKER, C. C. \& TAKAHASHI, E. M. 2001a. On the occurrence of testate amoebae (Protozoa, Rhizopoda) in Brazilian inland waters. III. Family Difflugidae: Genus Difflugia. Acta Scientiarum, Biological Sciences 23(2):305-321.

Lansac-Tôha, F. A.; Velho, L. F. M.; Takahashi, E. M.; Aoyagui, A. S. M. \& Bonecker, C. C. 2001b. On the occurrence of testate amoebae (Protozoa, Rhizopoda) in Brazilian inland waters V. Families Hyalospheniidae, Plagyopixidae, Microcoryciidae, Cryptodifflugiidae, Phryganelidae, Euglyphidae, Trinematiidae and Cyphoderiidae. Acta Scientiarum, Biological Sciences 23(2):333-347.

LansaC-Tôha, F. A.; Zimmermann-Callegari, M. C.; Alves, G. M.; Velho, L. F. M. \& Fulone, L. J. 2007. Species richness and geographic distribuition of testacea amoebae (Rhizopoda) in Brazilian inland freshwaters enviroments. Acta Scientiarum, Biological Sciences 29(2):185-195.

LansaC-Tôha, F. A.; Bonecker, C. C.; Velho, L. F. M.; Takahashi, E. M.; Joko, C. Y.; Alves, G. M.; Silva, N. R. S.; Fulone, L. J.; Leal, C. M.; Fernandes, A. P. C.; Costa, D. M.; SAntos, R. M.; Bastos, V. C.; Soares, P. A.; Braghin, L.; Nedlei, J. \& Lopez-Filho, D. R. 2008. In: Relatório Anual / PELD - Sítio 6 - A Planície Alagável do Alto Rio Paraná. Maringá, UEM/Nupélia/PELD/CNPq, p.123-156.

Lansac-Tôha, F. A.; Bonecker, C. C.; Velho, L. F. M.; Simões, N. R.; Dias, J. D.; Alves, G. M. \& Takahashi, E. M. 2009. Biodiversity of zooplankton communities in the Upper Paraná River floodplain interanual variation from long-term studies. Brazilian Journal of Biology 69(2):539-549.

LeIPNITZ, B.; LeÃo, C. \& Ferreira, F. 2005. Amebas testáceas em sedimentos quaternários do sistema lacustre de três Lagoas, MS. Gaea 1(2):82-93.

MADRID, J. Z. 2006. Estado de conocimiento de los tecamebianos dulceacuicolas de Chile. Gayana 70(1):27-30.

Medina Júnior, P. B. \& Rietzer, A. C. 2005. Limnological study of a Pantanal saline lake. Brazilian Journal of Biology 65(4):651-659.

Mizuta, S.; Imai, H.; Chang, K. H.; Doi, H.; Nishibe, Y. \& NaKano, S. 2011. Grazing on Microcystis (Cyanophyceae) by testate amoebae with special reference to cyanobacterial abundance and physiological state. Limnology 12(3):205-211.

Oliveira, M. T. \& Hardoim, E. L. 2010. Study of testacean assemblages (Protozoa: Rhizopoda) in touristic waterfall regions of Chapada dos Guimarães National Park, Mato Grosso State, Brazil. Acta Scientiarum, Biological Sciences 32(4):387-395.
Patterson, R. T.; Barker, T. \& Burdidge, S. 1996. Arcellaceans (thecamoebians) as proxis of arsenic and mercury contamination in northeastern Ontario lakes. Journal of Foraminiferal Research 26:172-183.

Pinto-Coelho, R. M. 2004. Métodos de coleta, preservação, contagem e determinação de biomassa de zooplâncton de águas epicontinentais. In: Bicudo, C.E.M. \& Bicudo, D.C. orgs. Amostragem em Limnologia. São Carlos, Rima, p.149-166.

Regali-Seleghim, M. H.; Godinho, M. J. L. \& Matsumura-Tundisi, T. 2011. Checklist dos "Protozoários" de água doce do estado de São Paulo, Brasil. Biota Neotropica 11(1a):135-172.

Reynolds, C. S.; Huszar, V.; KruK, C.; Naselli-Flores, L. \& Melo, S. 2002. Towards a functional classification of the freshwater phytoplankton. Journal of Plankton Research 24:417-428.

RochA, O. 2003. Organismos de Águas Doces. In: Lewinsohn, T. M. org. Avaliação do estado do conhecimento da diversidade biológica do Brasil. Brasília, MMA, p.13-52.

SANDERS, R.W. \& WickHAM, S. A. 1993. Planktonic protozoa and metazoan: predation, food quality and population control. Marine Microbial Food Webs 7(2):197-223.

SHERR, E. B. \& SHERR, B. F. 1994. Bacterivory and herbivory: key roles of phagotrophic protists in pelagic food webs. Microbial Ecology 28:223-235.

SiLVA, M. B. 2008. Assembléias de amebas testáceas (Amoebozoa: Rhizopoda) associadas à rizosfera de Eichhorniacrassipes (Martius) Solomons (Pontederiaceae) no rio Cachoeira, Bahia. Dissertação de Mestrado, Universidade Estadual de Santa Cruz, Ilhéus.

VelHo, L. F. M.; Bini, L. M. \& LANSAC-TôHA, F. A. 2004. Testate amoeba (Rhizopoda) diversity in plankton of the Upper Parana River floodplain, Brazil. Hydrobiologia 523:103-111.

VELHO, L. F. M. \& LANSAC-TôHA, F. A. 1996. Testate amoebae (Rhizopodea, Sarcodina) from zooplankton of the upper Paraná River floodplain, state of Mato Grosso do Sul, Brazil: II. Families Difflugidae. Studies on Neotropical Fauna and Environment 31:179-192.

Velho, L. F. M.; LanSaC-TôHA, F. A. \& BinI, L. M. 1999. Spatial and temporal variation in densities of testate amoebae in the plankton of the Upper Paraná River floodplain, Brazil. Hydrobiologia 411:103-113.

Velho, L. F. M.; Lansac-TôHa, F. A.; Bonecker, C. C. \& ZimmermannCAllegari, M. C. 2000. On the occurrence of testate amoebae (Protozoa, Rhizopoda) in Brazilian inland waters. II. Families Centropyxidae, Trigonopyxidae and Plagiopyxidae. Acta Scientiarum 22(2):365-374.

Velho, L. F. M.; Lansac-TôHa, F. A.; Bonecker, C. C.; ZimmermannCallegari, M. C. \& Aoyagui, A. S. M. 2001. On the occurrence of testate amoebae (Protozoa, Rhizopoda) in Brazilian inland waters. IV. Families Difflugiidae (genus Cucurbitella, Lagenodifflugia, Pentagonia, Pontigulasia, Protocucurbitella, Suiadifflugia) and Lesquereusiidae. Acta Scientiarum, Biological Sciences 23:323-332.

Velho, L. F. M.; Lansac-Tôha, F. A. \& Serafim-Junior, M. 1996. Testate amoebae (Rhizopodea-Sarcodina) from zooplankton of the upper Paraná river floodplain, state of Mato Grosso do Sul, Brazil. I. Families Arcellidae and Centropyxidae. Studies on Neotropical Fauna and Environment 31:35-50.

Velho, L. F. M.; Lansac-Tôha, F. A. \& Bini, L. M. 2003. Influence of environmental heterogeneity on the structure of testate amoebae (Protozoa, Rhizopoda) assemblages in the plankton of the Upper Paraná River floodplain, Brazil. International Review of Hydrobiology 88: $154-166$.

WAILES, G. H. 1913. Freshwater Rhizopoda from North and South America. Journal of the Linnean Society of London 32:201-218.

WALKER, I. 1982. The thecamoebae (Protozoa, Rhizopoda) of small Amazonian forest streams and their possible use as indicator organisms for water quality. Supl. Acta Amazonica 12(3):535-544. 